\title{
Predator-Prey Biogeography-Based Optimization for Bio-inspired Visual Attention
}

\author{
Xiaohua Wang \\ State Key Laboratory of Virtual Reality Technology and Systems, School of Automation Science and Electrical \\ Engineering, Beihang University \\ Beijing 100191, P R China \\ Haibin Duan* \\ 1. State Key Laboratory of Virtual Reality Technology and Systems, School of Automation Science and Electrical \\ Engineering, Beihang University \\ 2. Science and Technology on Aircraft Control Laboratory, Beihang University \\ Beijing 100191, P R China \\ E-mail: hbduan@buaa.edu.cn; 86-10-8231-7318 \\ Received 12 November 2012 \\ Accepted 15 May 2013
}

\begin{abstract}
Visual attention mechanism is one of the important techniques in computer vision field, and it can increase the effectiveness of computer image information processing. Biogeography-Based Optimization (BBO) is a bioinspired algorithm for global optimization, searching for the global optimum mainly through two steps: migration and mutation. In this paper, a novel Predator-Prey Biogeography-Based Optimization (PPBBO) is utilized to solve the bio-inspired visual attention problem. In PPBBO method, BBO is combined with the mechanism of predatorprey, which can enhance the global convergence of the algorithm. The convergence property of the PPBBO is analyzed theoretically, and the detailed process is also given. Comparative experimental results with basic BBO, CBBO, CPPBBO, and Particle Swarm Optimization (PSO) demonstrate the feasibility and effectiveness of our presented PPBBO for adjusting combination of feature maps in visual attention.
\end{abstract}

Keywords: Biogeography-Based Optimization (BBO), visual attention, saliency, feature combination, predatorprey.

\section{Introduction}

Human Visual System (HVS) can detect salient objects in complex scenes efficiently with the visual attention mechanism. A conceptually simple bio-inspired visual attention computational model simulating human visual attention has been proposed by Koch and Ullman ${ }^{1}$. This model consequently represents a complete account of bottom-up saliency and does not require any top-down guidance to shift attention ${ }^{2}$.
The whole visual attention process can be divided into two layers, visual information extracting and visual attention guiding. The input image is firstly decomposed into a set of topographic feature maps by extracting its intensity, color, and orientation. Afterwards the topographic feature maps are combined with the weighting coefficients into a saliency map. Finally the strategy of Winner-Take-All (WTA) is used to gain the most active location in this representation. To compute the weighting coefficients efficiently Predator-Prey 


\section{Biogeography-Based Optimization (PPBBO) is employed.}

BBO is a bio-inspired algorithm proposed by $\mathrm{Simon}^{3}$, which emulates the geographical distribution and the migration of species in an ecosystem. In this method each feasible solution is represented by a habitat. The Habitat Suitability Index (HSI) is introduced to measure the goodness of the habitat. BBO works mainly based on the two mechanisms: migration and mutation. With the migration mechanism, poor solutions can accept a lot of new features from good ones, which may improve the quality of those solutions. Furthermore, solutions do not have the tendency to clump together in similar groups due to the new type of mutation operation in BBO. Elitism operation ${ }^{4}$ can retain the best solutions in the population from one generation to the next and make the $\mathrm{BBO}$ algorithm more efficient.

The concept of predator-prey ${ }^{5}$ is introduced to BBO and these approaches are used to make an adjusted combination of feature maps in visual attention. Predator-prey is a strategy that chooses predators from the population of solutions to hunt the worst solutions and makes other solutions run away from those worst ones. It has been included in the PPBBO successfully in order to improve its capability of finding best solutions ${ }^{6}$. Comparative experiments have demonstrated that the PPBBO approach converges in less iterations when it is compared with the classical BBO. The simulation results show that the presented $\mathrm{PPBBO}$ is more competitive and manifests better efficiency.

The remainder of this paper is organized as follows. Section 2 presents the visual attention mechanism. Section 3 introduces the basic principle of BBO with elitism. In addition, this section presents the fitness function used in the algorithm while section 4 describes the principle of the concept of the predator-prey and give some theoretical analysis of the PPBBO. In section 5 the detailed implementation procedures of the PPBBO algorithm for optimizing the weighting coefficients are specified. In section 6, a series of comparative experiments are given to verify the effectiveness of the PPBBO approach. Our concluding remarks are contained in the final section.

\section{Bio-inspired Visual Attention}

\subsection{The principle of visual attention}

Visual attention is a mechanism which filters out redundant visual information and detects the most relevant parts of our visual field ${ }^{7}$. There are two kinds

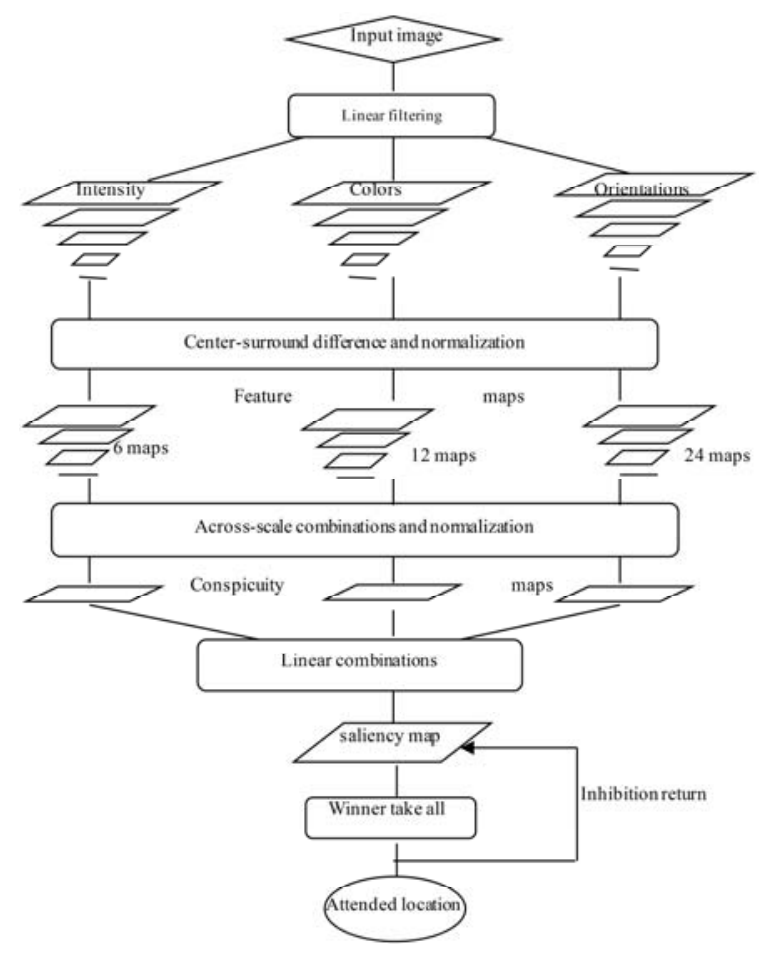

Fig.1 General structure of the visual attention model

of visual attention mechanisms, the bottom-up or stimulus-driven attention and the top-down or goaldriven attention. The model of bio-inspired visual attention mechanism (Fig. 1) is proposed by Koch and Ullman $^{1}$. This mechanism is rapid, bottom-up, saliencydriven, and task-independent. In this model, dyadic Gaussian pyramids are applied to create spatial scales and a set of linear center-surround operations, that are akin to visual receptive field, are used to compute each feature. This architecture is particularly well-suited to detecting locations which stand out from their surround as it is sensitive to local spatial discontinuities ${ }^{3}$. All the feature maps are combined into a saliency map with 
weight coefficients, the focus of attention are obtained according to the saliency map.

\subsection{Visual features extracting}

The HSI color space model is used for intensity features extracting, in order to reflect the color information better. With $r, g$ and $b$ presenting the red, green, and blue channels of the input image, an intensity image $I$ can be calculated with Eq. (1).

$$
I=\frac{(r+g+b)}{3}
$$

In the visual cortex, Color-Double-Opponent system is employed to present the visual sensitivity to color $^{8}$. In order to remove the coupling relationship between color channel and the intensity, channel $r, g, b$ is normalized into $r^{\prime}, g^{\prime}$ and $b^{\prime}$ with intensity $I$. Four broadly-tuned color channels are created with the Eq. (2).

$$
\left\{\begin{array}{l}
R=r^{\prime}-\left(g^{\prime}+b^{\prime}\right) / 2 \\
G=g^{\prime}-\left(r^{\prime}+b^{\prime}\right) / 2 \\
B=b^{\prime}-\left(r^{\prime}+g^{\prime}\right) / 2 \\
Y=\left(r^{\prime}+g^{\prime}\right) / 2-\left|r^{\prime}-g^{\prime}\right| / 2-b^{\prime}
\end{array}\right.
$$

Where $R, G, B$ and $Y$ for red, green, blue and yellow channels (negative values are set to zeros).

According to Color-Double-Opponent system, maps $R G$ are created in the model to simultaneously account for red/green and green/red double opponency and maps $B Y$ for blue/yellow and yellow/blue double opponency in Eq.(3).

$$
\left\{\begin{array}{l}
R G=R-G \\
B Y=B-Y
\end{array}\right.
$$

Local orientation information is obtained from $I$ with oriented Gabor pyramids $O(\theta)$, where $\theta \in\left\{0^{\circ}, 45^{\circ}, 90^{\circ}, 145^{\circ}\right\}$ is the preferred orientation.

For feature channels of the input image, structure Gaussian pyramids in eight octaves. Six Gaussian pyramids $I(\sigma), R(\sigma), G(\sigma), B(\sigma), Y(\sigma), O(\sigma, \theta)$

for intensity, red, green, blue, yellow and orientation can be obtained, where $\sigma \in[0,8]$, is the scale and $\theta \in\left\{0^{\circ}, 45^{\circ}, 90^{\circ}, 145^{\circ}\right\}$ is the orientation.

\subsection{The saliency map}

Each feature is computed by the linear center-surround operation that is similar to the function visual receptive field. Center-surround is based on a Difference of Gaussian (DOG), implemented in the model as the difference between fine and coarse scales. Specific operations are as following:

$$
\left\{\begin{array}{l}
I(c, s)=|I(c) \Theta I(s)| \\
R G(c, s)=|(R(c)-G(c)) \Theta(G(s)-R(s))| \\
B Y(c, s)=|(B(c)-Y(c)) \Theta(Y(s)-B(s))| \\
O(c, s, \theta)=|O(c, \theta) \Theta O(s, \theta)|
\end{array}\right.
$$

Where $\theta \in\left\{0^{\circ}, 45^{\circ}, 90^{\circ}, 145^{\circ}\right\}$, and $\Theta$ is the acrossscale difference between two maps $\mathrm{c}$ and $\mathrm{s}$. $c \in\{2,3,4\}, s=c+\delta, \delta \in\{3,4\}$. It is obvious that center-surround operations are carried out between maps 2-5, 2-6, 3-6, 3-7, 4-7 and 4-8.

The model obtains 42 feature maps in total: 6 intensity maps, 12 color maps and 24 orientation maps. A map normalization operator $N($.$) is used to get conspicuity$ maps with following operations at the $\sigma=4$ scale of the saliency map, with I for intensity, C for color and $\mathrm{O}$ for orientation. Where $\theta \in\left\{0^{\circ}, 45^{\circ}, 90^{\circ}, 145^{\circ}\right\}$, and 
$\Theta$ is the across-scale difference between two maps $c$

and s. $c \in\{2,3,4\}, s=c+\delta, \delta \in\{3,4\}$. It is obvious that center-surround operations are carried out between maps 2-5, 2-6, 3-6, 3-7, 4-7 and 4-8.

The model obtains 42 feature maps in total: 6 intensity maps, 12 color maps and 24 orientation maps. A map normalization operator $N($.$) is used to get conspicuity$ maps with following operations at the $\sigma=4$ scale of the saliency map, with $I$ for intensity, $C$ for color and $O$ for orientation.

$$
\left\{\begin{array}{l}
I(i)=N(I(c, s)) \quad i=1,2, \ldots, 6 \\
C(j)=N(R G(c, s) \cup N(B Y(c, s)) \quad j=1,2, \ldots, 12 \\
O(k)=N(O(c, s, \theta) \quad k=1,2, \ldots, 24
\end{array}\right.
$$

According to Eq. (6) the normalized conspicuity maps are summed into a saliency map.

$$
\begin{aligned}
S= & \frac{1}{\sum_{i=1}^{6} K_{1 i}+\sum_{j=1}^{12} K_{2 j}+\sum_{k=1}^{24} K_{3 k}} \times \\
& {\left[\sum_{i=1}^{6} K_{1 i} \times I(i)+\sum_{j=1}^{12} K_{2 j} \times C(j)+\sum_{k=1}^{24} K_{3 k} \times O(k)\right] }
\end{aligned}
$$

Where $K_{1 i}, K_{2 j}, K_{3 k}$ are the weight coefficients adjusted by intelligent algorithms, such as PPBBO algorithm presented in this work.

\section{Overview of the Biogeography-Based Optimization}

\subsection{Principles of $\mathrm{BBO}$ algorithm}

$\mathrm{BBO}$ is a stochastic optimization technique that is inspired by the geographical distribution of species and the migration of species in an ecosystem for solving multimodal optimization problems. The problem can be of any area in life as long as we have a qualitative measure of the suitability of a given solution ${ }^{9}$. In our paper, BBO technique is used to optimize the parameters of bio-inspired visual attention mechanism. In BBO, a set of habitats are used to present the possible solutions, and Suitability Index Variables(SIV) are used to describe the feature of each habitat while a HSI is the evaluation criteria to measure the goodness of a solution. BBO works mainly based on two mechanisms, migration and mutation, which help the species of less favorable area to acquire good features from the species in the favorable islands and strengthen the weak elements $^{10}$. Suppose that there have is a habitat $\mathrm{H}$, a vector of SIVs, following the migration and mutation steps to new candidate habitats are generated as shown in detail in following sections.

\subsection{The migration strategy}

The migration strategy is similar to the evolutionary strategy in which many parents can contribute to a single offspring ${ }^{11}$. Migration can be expressed as $H_{i}(S I V) \leftarrow H_{j}(S I V)^{12}$. Each individual has its own immigration rate $\lambda$ and emigration rate $\mu$, which can be calculated as following when there are $S$ species in the habitat:

$$
\left\{\begin{array}{l}
\mu_{\mathrm{s}}=\frac{\mathrm{ES}}{S_{\max }} \\
\lambda_{\mathrm{s}}=I\left(1-\frac{S}{S_{\max }}\right)
\end{array}\right.
$$


Where $E$ is the maximum emigration rate, and $I$ is the maximum immigration rate, while $S_{\max }$ is the largest possible number of species that the habitat can support. The immigration and emigration curves are shown in Fig. 2.

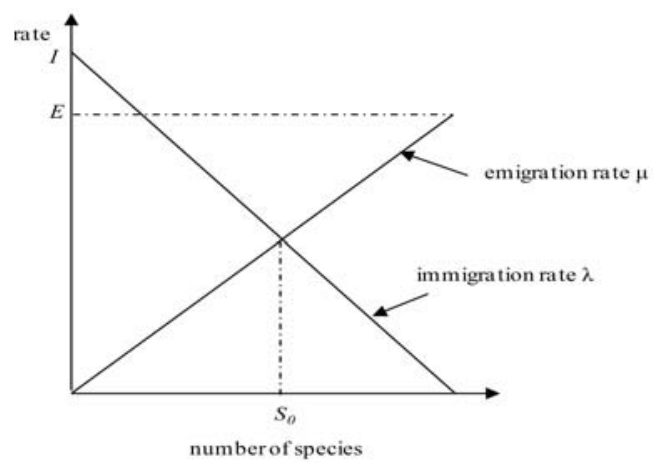

Fig. 2. Immigration and emigration curves of BBO

\subsection{The mutation strategy}

As HSI of a habitat can change suddenly due to apparently random events, in BBO there is SIV mutation. Species count probabilities is used to determine mutation rates. $P_{s}$ is the probability that the habitat contains exactly $S$ species, and it changes from time $t$ to time $t+\Delta t$ as follows:

$$
P_{s}(t+\Delta t)=P_{s}(t)\left(1-\lambda_{s} \Delta t-\mu_{s} \Delta t\right)+P_{s-1} \lambda_{s-1} \Delta t+P_{s+1} \mu_{s+1} \Delta t
$$

Mutation is used to enhance the diversity of the population, which helps to decrease the chances of getting trapped in local optima. The mutation rate $m(s)$ is given in the following function proportional to $P_{s}$ :

$$
m(s)=m_{\max }\left(1-\frac{\mathrm{P}_{s}}{\mathrm{P}_{\max }}\right)
$$

Where $m_{\max }$ is a user-defined parameter, and $P_{\max }$ is the maximum species count probability, while $P_{S}$ is the probability of existence of $S$ species in the habitat.

This mutation scheme can increase diversity of the populations. On the other hand, with the elitism approach, which can save the features of the habitat that has the best solution in the $\mathrm{BBO}$ process, we can revert back to it even if mutation ruins its HSI.

\section{Predator-Prey Biogeography-Based Optimization}

\subsection{The predator-prey mechanism}

Predatory behavior is one of the most common phenomena in nature. Predators hunt prey to guarantee their own existence. The preys need to try their best to run away from predators. The concept of predator-prey has been introduced to $\mathrm{BBO}$ to increase the diversity of the population and overcome the problem of local optimum traps successfully. In the PPBBO algorithm, the model based on the worst solutions is used to choose the predators.

$$
S_{\text {predator }}=S_{\text {worst }}+\rho\left(1-k / k_{\max }\right)
$$

Where $S_{\text {predator }}$ is a possible solution chosen as a predator, and $S_{\text {worst }}$ is the worst solution in the population. $k$ is the number of current iteration, and $k_{\max }$ is the maximum number of the whole iterations, while $\rho$ is the hunting rate which is a certain number given. Eq. (11) is a model that describes the prey fleeing and provides the solutions to maintain a distance from the predator.

$$
\left\{\begin{array}{l}
S_{k+1}=S_{k}+\rho e^{-|d|}, d>0 \\
S_{k+1}=S_{k}-\rho e^{-|d|}, d<0
\end{array}\right.
$$

Where $d$ is the distance between the solution and the predator, and $k$ is number of current iteration. The combination of BBO and predator-prey can prevent the population from converging to a point in the search space and improve the capability of exploration evidently. Predator-prey mechanism is a prominent aspect which is introduced to enhance the diversity of the population and make the populations maintain a distance from the worse solutions. Obviously, this strategy can improve the efficiency of the algorithm.

\subsection{Theoretical analysis of $P$ PBBO}

A Markov chain model has been developed for basic BBO's selection, migration, and mutation operators ${ }^{13}$. The distribution of a BBO population can be represented by the Markov state. Suppose that the population size is $N$ and the possible solution are represented by $x_{i}$ consisting of $q$ dimensions. If the 
range of the solution is $r$, the cardinality of the search space is $S=r^{q}$. $v$ denotes the population vector, and $v_{i}$ is the number of $x_{i}$ individuals in the population. $x_{i}(s)$ is the $s$-th dimension of $x_{i}$, and $J_{i}(s)=\left\{j: x_{j}(s)=x_{i}(s)\right\}$.

The Markov model of the migration ${ }^{13}$ can be expressed as:

$$
\begin{gathered}
\operatorname{Pr}\left(y_{k}(s)_{t+1}=x_{i}(s)\right)=\left(1-\lambda_{m(k)}\right) 1_{0}\left(x_{m(k)}(s)-x_{i}(s)\right) \\
+\lambda_{m(k)} \frac{\sum_{j \in J_{i}(s)} v_{j} u_{j}}{\sum_{j=1}^{n} v_{j} u_{j}}
\end{gathered}
$$

Where $\lambda_{m(k)}$ is the probability of immigration

to $y_{k}(s)$, and $1_{0}$ is the indicator function of the set $\{0\}$.

$$
P_{k i}(v)=\operatorname{Pr}\left(y_{k . t+1}=x_{i}\right)=\prod_{s=1}^{q} \operatorname{Pr}\left(y_{k}(s)_{t+1}=x_{i}(s)\right)
$$

Where $P_{k i}(v)$ denotes the probability that immigration results in $y_{k}=x_{i}, q$ is the dimension of the solution.

$$
\begin{gathered}
\operatorname{Pr}(u \mid v)=\sum_{J \in Y} \prod_{k=1}^{N} \prod_{i=1}^{n}\left[P_{k i}(v)\right]^{J_{k i}}, \\
Y=\left\{J \in R^{N \times n}: J_{k i} \in\{0,1\},\right. \\
\left.\sum_{i=1}^{n} J_{k i}=1 \text { for all } k, \sum_{k=1}^{N} J_{k i}=u_{i} \text { for all } i\right\}
\end{gathered}
$$

Where $\operatorname{Pr}(u \mid v)$ is the probability that a population

vector, $u$ is obtained after a generation from $v$.

For the mutation strategy we use $U_{i j}$ to denote the

$$
P_{k i}^{(2)}(v)=\sum_{j=1}^{n} U_{i j} P_{k i}(v), P^{(2)}(v)=P(v) U^{T}
$$

Where $P_{k i}^{(2)}(v)$ denotes the probability that the mutation following the $k$-th immigration results in $X_{i}$.

$$
\operatorname{Pr}^{(2)}(u \mid v)=\sum_{J \in Y} \prod_{k=1}^{N} \prod_{i=1}^{n}\left[P_{k i}^{(2)}(v)\right]^{J_{k i}}
$$

For the predator-prey mechanism, $V_{i j}$ is used to denote the probability that $X_{j}$ flees to $X_{i}$.

$$
P_{k i}^{(3)}(v)=\sum_{j=1}^{n} V_{i j} P_{k i}^{(2)}(v), P^{(3)}(v)=P^{(2)}(v) V^{T}
$$

Where $P_{k i}^{(3)}(v)$ is the probability that the predator-prey following the $k$-th immigration and mutation results in $X_{i}$.

From the above analysis, it is obvious that the new population is merely relevant to current population, which means that $\{x(n), n \in N\}$ is a discrete time Markov chain.

The global optimal solution set can be expressed with $M=\max \left(f\left(x_{k}\right), k=1,2, \ldots, S\right)$, where $S$ is the cardinality of the search space.

Lemma1.The evolution direction of the habitat of PPBBO is unchangeable. i.e. $f\left(x_{i+1}\right) \geq f\left(x_{i}\right)$.

probability that $X_{j}$ mutates to $X_{i}$. 
Proof. In each generation, the greedy selection operator is used to choose a better solution and save the vector of the relevant habitat. At the same time elitism approach can save the features of the habitat that has the best solution in the BBO process, the beat solution can be reverted even if migration or mutation ruins the HSI.

Theorem 1. PPBBO algorithm converges to the global

optimal solution set $M \neq \varnothing$ in probability 1 , i.e. $\lim _{n \rightarrow \infty} P(x(n) \in M)=1$, which is independent with the initial distribution.

Proof. From Lemma1, it is obvious that

$$
P(x(n+1) \in M \mid x(n) \in M)=1
$$

Suppose that

$P(x(n+1) \in M \mid x(n) \notin M)=p(n)>0$, which is

tenable for most of the actual situation. The probability

that no population entered the global optimal solution

set after $n$ iterations $P_{\text {not }}(n)$ can be calculated as

following $^{14}$ :

$$
P_{n o t}(n)=\prod_{t=1}^{n}(1-p(t))
$$

Then

$$
P(x(n) \in M)=1-P_{n o t}(n)
$$

Suppose $n \rightarrow \infty$, then

$$
\begin{aligned}
& \lim _{n \rightarrow \infty} P(x(n) \in M)=1-\prod_{t=1}^{\infty}(1-p(t)) \\
& \text { As } 0<p(t) \leq 1 \text {, then } 0<1-p(t) \leq 1 .
\end{aligned}
$$

Thus

$$
\begin{gathered}
\prod_{t=1}^{\infty}(1-p(t))=0 \\
\lim _{n \rightarrow \infty} P(x(n) \in M)=1-\prod_{t=1}^{\infty}(1-p(t))=1
\end{gathered}
$$

The proof process presents that the establishment of the conclusion is independent of the initial population. Therefore, the PPBBO algorithm can converge to the global optimal solution set $M \neq \varnothing$.

\section{Our Visual Attention Based on PPBBO}

In this section, PPBBO is applied to adjust the combination weight coefficients of the feature maps in visual attention. The fitness function is defined to calculate the HSI of every habitat in different situations of the problems. Therefore, the normalized saliency of a given location is selected as the HSI, and the weight coefficients $K_{1 i}, K_{2 j}, K_{3 k}$ as shown in Eq. (6) are chosen as habitats. It is obvious that the fitness function is a 42 dimension optimization problem. The main steps involved in this process are given below:

Step 1: Image pre-processing. Obtain the original image and filter the image to remove the noise.

Step 2: Extract early visual features such as intensity, color and orientation with Eq.s (1)-(3). A Gaussian pyramid for each channel can be created, which is illustrated in section 2.2.

Step 3: Compute each feature is with a set of linear center-surround operations described in Eq. (4). Normalize the conspicuity maps with Eq. (5).

Step 4: Initialize the BBO parameters according to the optimizing problem. Derive a method of mapping problem solutions to SIVs and habitats, which is problem dependent. Initialize the maximum species count $S_{\max }$ and the maximum migration rates $E$ and $I$, the maximum mutation rate $m_{\max }$, and an elitism parameter Keep. Initialize the step size used for numerical 
integration of probabilities $d t$.

Step 5: Initialize habitats. Randomly initialize a set of habitats, each habitat corresponding to a potential solution to the optimizing problem of visual attention.

Step 6: Calculate and $\mu$. For each habitat, map the HSI to the number of species $S$, the immigration rate $\lambda$, and the emigration rate $\mu$ according to Eq. (7).

Step 7: Migrate. Use immigration rate $\lambda_{i}$ and emigration rate $\mu_{i}$ to probabilistically modify each nonelite habitat, then compute each HSI according to Eq. (6) again.

Step 8: Mutate. For each habitat, update the probability of its species count according to Eq. (8). Mutate each non-elite habitat based on its probability, and recompute each HSI according to the fitness function of PPBBO according to Eq. (6).

Step 10: Predator-prey mechanism. Find the habitat with the lowest HSI and use the Eq. (10) and (11) to hunt the solutions and re-compute HSI for each habitat according to Eq. (6).

Step 11:If the stopping criterion is satisfied, go to Step 12, otherwise, go to Step 6. This loop can be terminated after a number of iterations, or after an acceptable problem solution has been found.

Step 12:Use the best solution to create a saliency map from the feature maps, and output the optimized result.

The flow chart of the proposed hybrid algorithm can be shown in Fig. 3.

\section{Experimental Results}

In order to verify the feasibility and effectiveness of the PPBBO algorithm for visual attention, a set of comparative experiments are conducted in this section. The methods are implemented on a PC with $512 \mathrm{Mb}$ of RAM using Windows XP and are encoded in Matlab 2010. Based on the tests and practical experience, the initial parameters of different methods are set as following. The number of the dimensions of the problem is decided by the number of the weighting coefficients, that is 42 in the mechanism described in section 2.3. Population size $P=50$, number of generations $N=100$, step size used for numerical integration of probabilities $d t=1$, the maximum migration rates $I=1, E=1$, mutation probability $m=0.03$, the number of elitisms keep $=3$ and the adopted hunting rate $\rho=0.02$ for the PPBBO approach. As shown in Fig.4 (a), there are three sailboats in the scene. PPBBO is employed to optimize the visual attention to make the red one stand out in the saliency map. The results are shown in Fig.4.

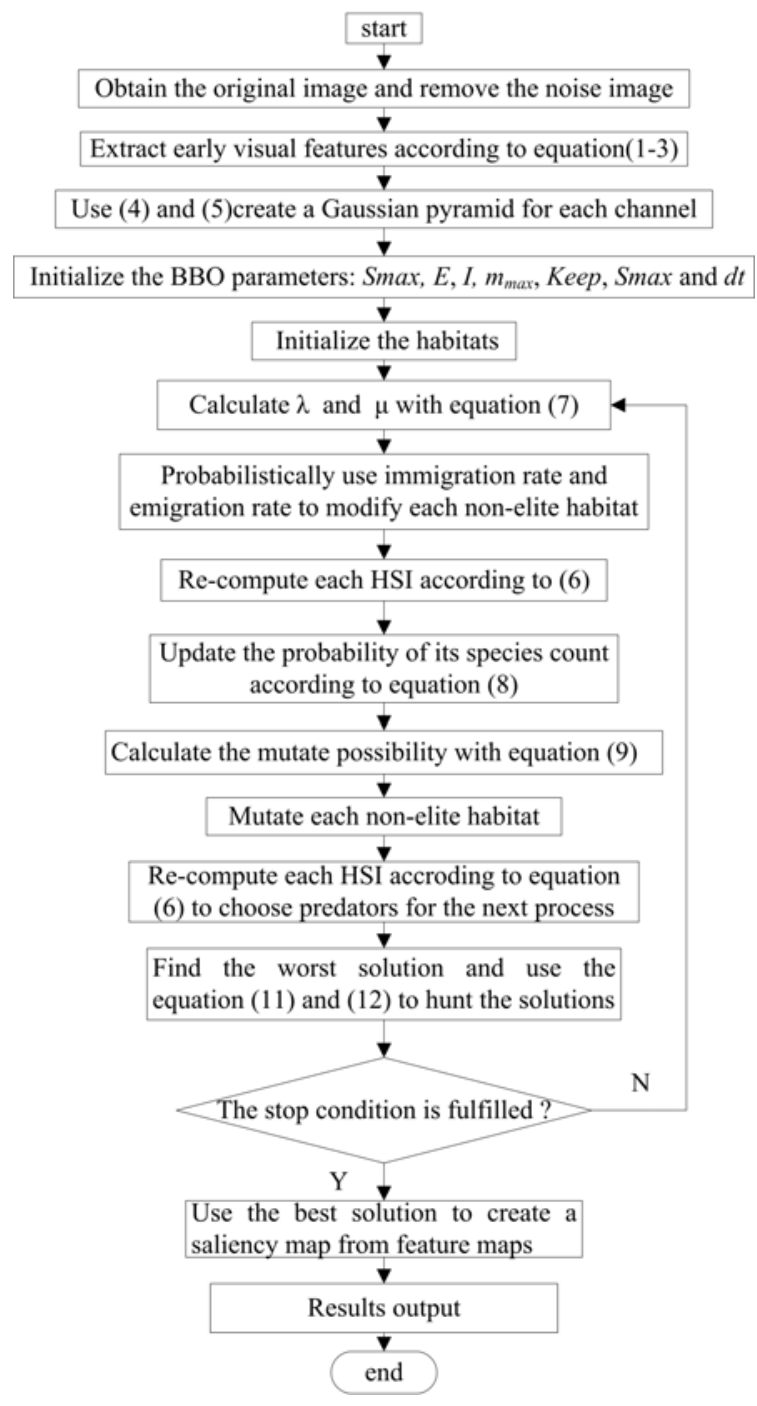

Fig.3. Flowchart of PPBBO for visual attention 


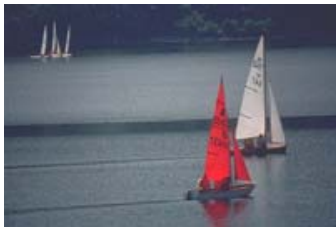

(a)

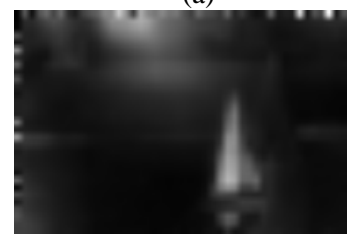

(c)

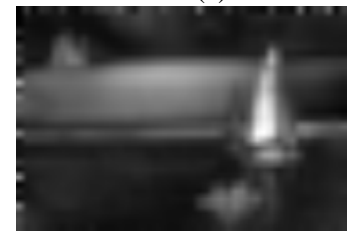

(e)

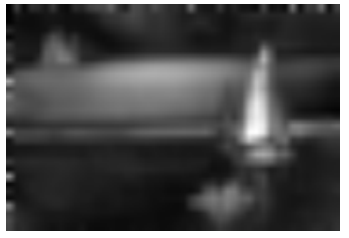

(b)

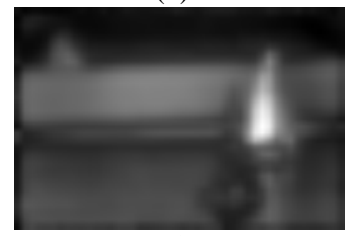

(d)

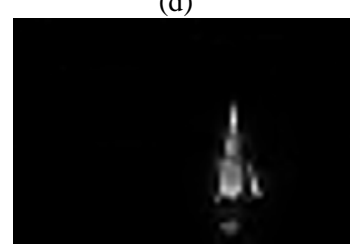

(f)
Fig.4 Experimental results for Case 1.(a)Original picture; (b) Intensity feature map;(c) Color feature map; (d) Orientation feature map;(e) Saliency map without optimization; (f) Saliency map with PPBBO.

Feature maps of 3channels are shown in Fig.4 (b), Fig.4(c), and Fig.4 (d). From the experiment results given in Fig.4 (e), it is obvious that the basic visual attention can make the salient origins stand out from others. However, the focus of attention is not very clear as there is not only one salient location. The visual attention is not that satisfying especially when a certain location for example, the red sailboat in Fig.4 (a), is expected to stand out. However, the presented PPBBO can efficiently make better performance as shown in Fig.4 (f). One more complex case is given in Fig. 5 to enhance the function of PPBBO optimization. In this case, the white architectures are expected to be highlighted, and the results are given in Fig. 5.

Fig. 4 and Fig. 5 show that the effective PPBBO method can successfully make the expected origin salient. Further experiments are given in Fig.6 and Fig.7 to verify the stability and advantage of the PPBBO. For each case, the algorithm runs for 10 times independently and the evolution curves are obtained to test if the

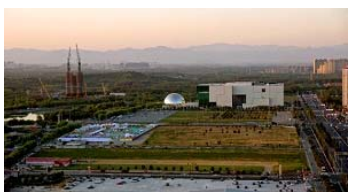

(a)

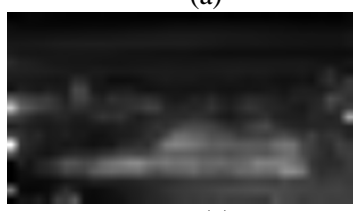

(c)

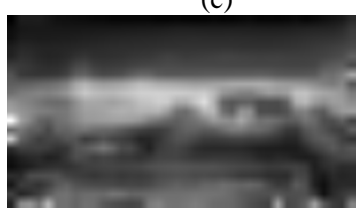

(e)

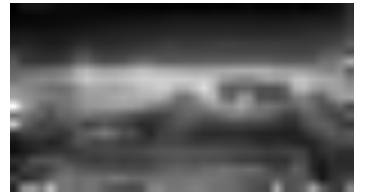

(b)

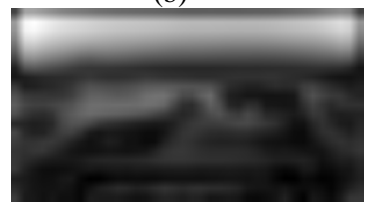

(d)

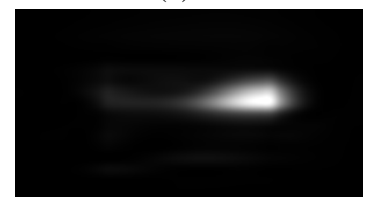

(f)
Fig.5 Experimental results for Case 2.(a)Original picture; (b) Intensity feature map;(c) Color feature map; (d) Orientation feature map;(e) Saliency map without optimization ;(f) Saliency map with PPBBO.

algorithm is stable. As bio-inspired intelligence is in the spotlight in the field of international artificial intelligence, numbers of bio-inspired intelligent methods have emerged in recent years ${ }^{15,16}$. To prove that the performance of PPBBO is better than others, some competitive bio-inspired intelligent methods should be chosen.

Chaos is a general nonlinear phenomenon in nature, and its behavior is nearly stochastic ${ }^{17}$. Chaotic behavior has already been observed in the laboratory in a variety of systems including electrical circuits, lasers, oscillating chemical reactions, fluid dynamics, as well as computer models of chaotic processes ${ }^{18}$. The chaos theory is introduced to BBO (CBBO) and PPBBO (CPPBBO) for optimizing the search process. The optimized results of PPBBO, CBBO, CPPBBO, basic BBO and the Particle Swarm Optimization (PSO) ${ }^{17}$ methods are presented in Fig.6 and Fig.7. The population sizes of all these algorithms are 50.The numbers of iterations for PPBBO, CPPBBO, and CBBO are all set to 50 , and that for the basic BBO is set to 100 . As the performance of PSO is worse, the iterative time of it is set to150. 


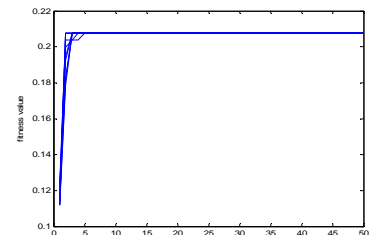

(a)

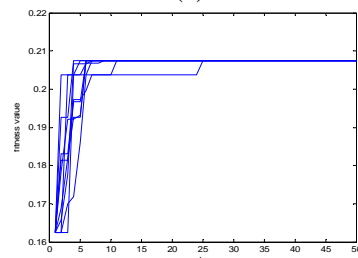

(c)

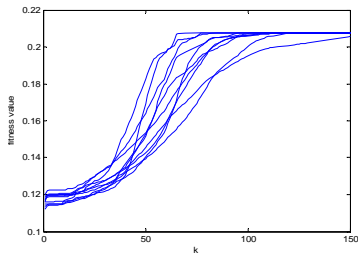

(e)

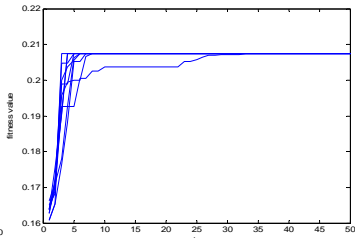

(b)

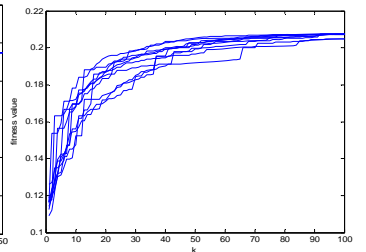

(d)

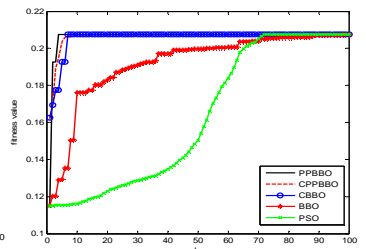

(f)
Fig.6.Comparative results for case 1. (a)Evolution curves of PPBBO;(b) Evolution curves by of CPPBBO;(c)Evolution curves of CBBO;(d) Evolution curves of BBO;(e) Evolution curves of PSO;(f). Evolution curves in comparison.

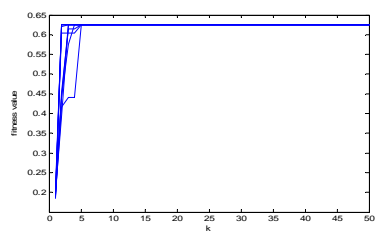

(a)

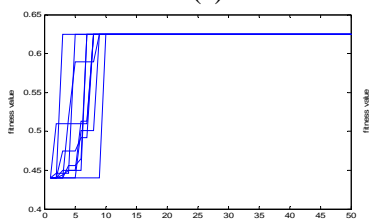

(c)

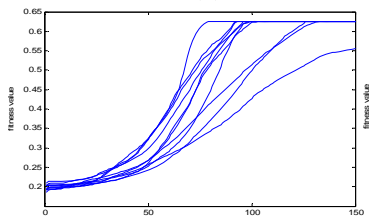

(e)

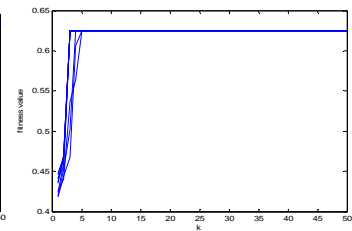

(b)

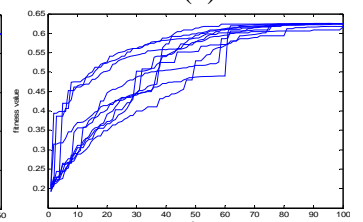

(d)

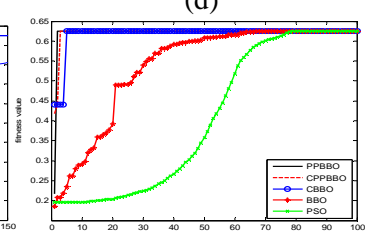

(f)
Fig.7.Comparative results for case 2. (a)Evolution curves of PPBBO;(b) Evolution curves by of CPPBBO;(c)Evolution curves of $\mathrm{CBBO}$;(d) Evolution curves of BBO;(e) Evolution curves of PSO;(f). Evolution curves in comparison.
In Fig.6 and Fig.7, the evolution curves of the PPBBO, СРPBBO, СBBO, BBO and PSO are all presented. Among them, the converging rate of PPBBO is the fastest apparently. PPBBO finds the best solution at the 3th iteration on average while CPPBBO needs 4th iteration. CBBO needs 5th iteration, and the other two algorithms need much more iterations. Superficially, the converging rate of $\mathrm{PPBBO}, \mathrm{CPPBBO}$, and $\mathrm{CBBO}$ are almost the same. PPBBO is much more competitive when we take the time cost into consideration, which is a very important criterion especially to real-time tasks. To further highlight the advantages of the method, the statistical performances of 50 independent runs of each method are listed in Table 1.

From Table 1, it is obvious that in case 1 the average converged iteration number of our PPBBO algorithm is 3 , while $\mathrm{CPPBBO}$ algorithm needs one generation more than it and CBBO algorithm needs two more. It seems that there is no obvious advantage that our algorithm has when it is compared with the other two algorithms in this aspect. However, it also shows that our method spends less time than CPPBBO and CBBO regardless of whether average or convergent time cost. The standard $\mathrm{BBO}$ and PSO need less exhaustive computing time but they converge much slower than the other three methods. In fact, the convergent speed of our method PPBBO is much faster than the others that means if only considering of the successful runs, the time cost of PPBBO to find the best solution is much less than the other 4 methods as shown in the table. It is very apparent when considering the real-time criteria, our presented PPBBO performs much better for optimizing the parameters to combine a saliency map in visual attention.

\section{Conclusions}

This paper presented a PPBBO algorithm for optimizing the parameters in the process of bio-inspired visual attention. Bio-inspired visual attention is of great importance to overcome the current existing problems of poor real-time performance and low accuracy in the extraction of interesting objects and other image processing areas. The bio-inspired and population-based optimization technique $\mathrm{BBO}$ is a new global optimization algorithm that has shown a good performance. Utilizing the mechanism of predator-prey allows PPBBO to be more stable and faster converging. Comparative experimental results of $\mathrm{BBO}$, $\mathrm{CBBO}$, 
Table 1. Comparative results by five different algorithms

\begin{tabular}{|c|c|c|c|c|c|c|}
\hline \multicolumn{2}{|c|}{ Compared parameters } & PPBBO & СРРВBО & CBBO & $\mathrm{BBO}$ & PSO \\
\hline Average converged & Case 1 & 3 & 4 & 5 & 67 & 98 \\
\hline Iteration & Case2 & 3 & 4 & 5 & 72 & 102 \\
\hline Average CPU time & Case 1 & $2.6346 \mathrm{~s}$ & $5.3967 \mathrm{~s}$ & $4.0487 \mathrm{~s}$ & $1.3272 \mathrm{~s}$ & $1.1253 \mathrm{~s}$ \\
\hline (one iteration) & Case2 & $3.1062 \mathrm{~s}$ & $6.2337 \mathrm{~s}$ & $4.7167 \mathrm{~s}$ & $1.5293 \mathrm{~s}$ & $1.3326 \mathrm{~s}$ \\
\hline \multirow{2}{*}{ Convergent CPU time } & Case 1 & $7.9038 \mathrm{~s}$ & $21.5868 \mathrm{~s}$ & $24.9348 \mathrm{~s}$ & $88.9224 s$ & $110.2794 \mathrm{~s}$ \\
\hline & Case2 & 9.3186 & $24.9348 \mathrm{~s}$ & $20.2435 \mathrm{~s}$ & $109.7208 \mathrm{~s}$ & $135.9252 \mathrm{~s}$ \\
\hline
\end{tabular}

CPPBBO and PSO are also given to verify the feasibility and effectiveness of the PPBBO approach. It may finally be concluded that PPBBO is a more effective and robust method of combining feature maps in the bio-inspired visual attention process.

Our future work will focus on improving the migration rates and parameter selection strategy of PPBBO. More feature maps and other factors should be further investigated to make the focus of attention more accurate.

\section{Acknowledgements}

This work was partially supported by Natural Science Foundation of China (NSFC) under grant \#61273054, \#60975072 and \#60604009, National Key Basic Research Program of China under grant \#2013CB035503, Program for New Century Excellent Talents in University of China under grant \#NCET-10-0021, Top-Notch Young Talents Program of China, Graduate Innovation Foundation for Beihang University under grant \#YCSJ-01-201206, and Aeronautical Foundation of China under grant \#20115151019.

\section{References}

1. Christof Koch, Shimon Ullman. Shifts in selective visual attention: towards the underlying neural circuitry. Human Neurobiology, 4(4) (1985) 219-227. DOI: 10.1007/97894-009-3833-5_5.

2. Laurent Itti, Christof Koch, Ernst Niebu. A model of saliency-based visual attention for rapid scene analysis. IEEE Transactions on Pattern Analysis and Machine Intelligence. 20(11) (1998) 1254-1259. DOI: $10.1109 / 34.730558$.
3. Dan Simon. Biogeography-based optimization. IEEE Transactions on Evolutionary Computation. 12(6) (2008) 702-713. DOI: 10.1109/TEVC.2008.919004.

4. Dan Simon, Mehmet Ergezer, Dawei Du. Population distributions in bio-geography-based optimization algorithms with elitism. Proceedings of the IEEE International Conference on Systems, Man, and Cybernetics( San Antonio, TX, USA. 2009), 991-996. DOI: 10.1109/ICSMC.2009.5346058.

5. Arlindo Silva, Ana Neves, Ernesto Costa. SAPPO. A simple, adaptable,predator prey optimiser. In Lecture Notes in Computer Science (EPIA 2003)(Heidelberg, Germany Springer-Verlag 2003) 2902, 59-73. DOI: 10.1007/978-3-540-24580-3_14.

6. Marsil de A. Costa e Silva, Leandro dos S. Coelho, and Luiz Lebensztajn. Multiobjective biogeography-based optimization based on predator-prey approach. IEEE Transactions on Magnetics. 48(2) (2012) 951-954. DOI: 10.1109/TMAG.2011.2174205.

7. Olivier Le Meur, Patrick Le Callet, Dominique Barba, Dominique Thoreau. A coherent computational approach to model bottom-up visual attention. IEEE Transactions on Pattern Analysis and Machine Intelligence. 28(5) (2006) 802-817. DOI: 10.1109/TPAMI.2006.86.

8. Stephen Engel, Xuemei Zhang and Brian Wandell. Colour tuning in human visual cortex measured with functional magnetic resonance imaging. Nature. 388(6637) (1997) 68-71. DOI: 10.1038/40398.

9. K. Jamuna, K.S Swarup. Biogeography based optimization for optimal meter placement for security constrained state estimation. Swarm and Evolutionary Computation. 1(2) (2011) 89-96. DOI: 10.1016/j.swevo.2011.05.002.

10. S. Rajasomashekar, P. Aravindhababu. Biogeography based optimization technique for best compromise solution of economic emission dispatch. Swarm and Evolutionary Computation. 7 (2012) 1-1. DOI: 10.1016/j.swevo.2012.06.001. 
11. Li Xiangtao,Wang Jinyan, Zhou Junping, Yin Minghao. A perturb biogeography based optimization with mutation for globalnumerical optimization. Applied Mathematics and Computation. 218(2) (2011) 598-609. DOI: 10.1016/j.amc.2011.05.110.

12. Ma Haiping, Dan Simon. Blended biogeography-based optimization for constrained optimization. Engineering Applications of Artificial Intelligence. 24(3) (2011) 517525. DOI: 10.1016/j.engappai.2010.08.005.

13. Dan Simon, Mehmet Ergezer, Dawei Du. Markov models for biogeography-based optimization and genetic algorithms with global uniform recombination. IEEE Transactions on Systems, Man, and Cybernetics-Part B: Cybernetics. 41(1) (2011) 299-306. DOI: 10.1109/TSMCB.2010.2051149.

14. Yan Ling, Jiang Jingping. Convergence and escape capacity research of evolution learning strategies. Acta Automatica Sinica. 31(6) (2005) 873-880.

15. Duan HaiBin, Shao Shan, Su BingWei, Zhang Lei. New development thoughts on the bio-inspired intelligence based control for unmanned combat aerial vehicle. Science China Technological Sciences. 53(8) (2010) 2025-2031. DOI: 10.1007/s11431-010-3160-z.

16. Sun Changhao, Duan Haibin. Artificial bee colony optimized controller for MAV pendulum. Aircraft Engineering and Aerospace Technology. 85(2) (2013) 104-114. DOI: 10.1108/00022661311302715.

17. Liu Fang, Duan Haibin, Deng Yimin. A chaotic quantumbehaved particle swarm optimization based on lateral inhibition for image matching. Optik. 123(21) (2012) 1955-1960. DOI: 10.1016/j.ijleo.2011.09.052.

18. Xu Chunfang, Duan Haibin, Liu Fang. Chaotic artificial bee colony approach to uninhabited combat air vehicle (UCAV) path planning. Aerospace Science and Technology. 14(8) (2010) 535-541. DOI: 10.1016/j.ast.2010.04.008. 